\title{
Synthesis and characterization of polypyrrole doped with anionic spherical polyelectrolyte brushes
}

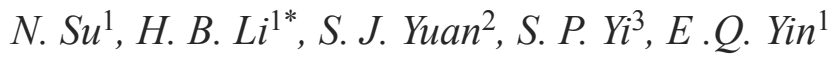 \\ ${ }^{1}$ School of Printing and Packaging, Wuhan University, 430079 Wuhan, China \\ ${ }^{2}$ School of Chemical and Environmental Engineering, Hubei University of Technology, 430068 Wuhan, China \\ ${ }^{3}$ College of Chemistry and Molecular Sciences, Wuhan University, 430072 Wuhan, China
}

Received 28 December 2011; accepted in revised form 16 March 2012

\begin{abstract}
The procedures for the synthesis of polypyrrole (PPy) doped with anionic spherical polyelectrolyte brushes (ASPB) (PPy/ASPB nanocomposite) by means of in situ chemical oxidative polymerization were presented. Fourier transform infrared spectroscopy (FTIR) and Raman spectroscopic analysis suggested the bonding structure of PPy/ASPB nanocomposite. Scanning electron microscopy (SEM) was used to confirm the morphologies of samples. The crystallographic structure, chemical nature and thermal stability of conducting polymers were analyzed by X-ray diffraction (XRD), X-ray photoelectron spectroscopy (XPS) and Thermo-gravimetric analysis (TGA) respectively. Investigation of the electrical conductivity at room temperature showed that the electrical conductivity of PPy/ASPB nanocomposite was $20 \mathrm{~S} / \mathrm{cm}$, which was higher than that of PPy $(3.6 \mathrm{~S} / \mathrm{cm})$.
\end{abstract}

Keywords: nanocomposites, conducting polymers, anionic spherical polyelectrolyte brushes, doping, electrical conductivity

\section{Introduction}

Since the first paper of Shirakawa et al. [1] in 1977, conducting polymers have attracted considerable interest because of their excellent physical and chemical properties originating from their unique $\pi$ conjugated system [2]. Polypyrrole (PPy) is one of the most extensively studied materials due to its easy synthesis and good environmental stability [3, 4]. However, pristine PPy is insoluble and infusible because of the rigidity of its molecular chains of $\pi$ conjugated structure. This poor conductivity and processibility not only impede further investigation of its structure, but also limit its application in technology. So efforts to overcome these drawbacks have led to numerous studies on the synthesis of PPy by both electrochemical and chemical routes. It is well-known that using various dopants has contributed to the development of soluble PPy [5]. Cur- rently, aromatic acid derivatives and organic sulfonates are widely used, such as dodecylbenzene sulfonic acid (DBSA), p-toluenesulfonic acid (TsOH) [6], etc. Lee et al. [7] developed DBSA with surface-active properties as anionic additive to realize pyrrole (Py) solvation. Further studies pointed out that adding other large dopants could reduce the interchain links, resulting in the increase of the conductivity and solubility [8-11]. In view of this, anionic spherical polyelectrolyte brushes (ASPB), consisting of anion polyelectrolyte chains affixing to the surface of spheres, may be novel conducting polymer dopant by introducing the brush polymer with certain functional groups.

The investigations on the structure and properties of PPy have been performed by many techniques. X-ray photoelectron spectroscopy (XPS) proved to be particularly useful in the surface characterization of

\footnotetext{
${ }^{*}$ Corresponding author, e-mail: 1 hb@whu.edu.cn
}

(c) BME-PT 
PPy [12-14] because it has a typical sampling depth of $2-10 \mathrm{~nm}$. Fourier transform infrared spectroscopy (FTIR) is a convenient technique to monitor changes in the structure of conducting polymer chains [15, 16]. Both experimental methods [17] and theoretical model [18] have been developed to describe the dependence of conductivity on conjugation length for PPy from FTIR measurements. In addition, Raman spectra [6], X-ray diffraction (XRD) [19], and Thermo-gravimetric analysis (TGA) [20] are also used to study the structure and properties of PPy.

In this paper, we presented a facile method for the synthesis of PPy doped with ASPB (PPy/ASPB nanocomposite) by in situ chemical oxidative polymerization [21, 22]. For comparison, PPy was synthesized first. The morphology of the resulting PPy/ ASPB nanocomposite was characterized by scanning electron microscopy (SEM). Information about its structure was obtained from FTIR, Raman spectra, XRD techniques, and its surface chemical composition by XPS measurements. The room temperature electrical conductivities and thermal stability of PPy and PPy/ASPB nanocomposite were investigated by a four-point probe apparatus and TGA respectively.

\section{Experimental}

\subsection{Materials}

Pyrrole (Sinopharm of Chemical Reagents Co., Ltd, Shanghai, China) was distilled under reduced pressure before use. Iron (III) chloride hexahydrate $\left(\mathrm{FeCl}_{3} \cdot 6 \mathrm{H}_{2} \mathrm{O}\right)$ and ethanol were purchased from Sinopharm of Chemical Reagents Co., Ltd, Shanghai, China. They were analytical reagents and were used without further purification. The ASPB $\left(D_{\mathrm{z}} \approx\right.$ $\left.100 \mathrm{~nm}, M_{\mathrm{w}}=1800 \mathrm{~g} / \mathrm{mol}, M_{\mathrm{w}} / M_{\mathrm{n}}=1.6\right)$ consisting of modified $\mathrm{SiO}_{2}$ cores and poly(Sodium-p-Styrenesulfonate) (PSS) brushes were prepared by surface-initiated polymerization.

\subsection{Synthesis of PPy/ASPB nanocomposite}

The synthesis of PPy/ASPB nanocomposite mainly involved: $0.22 \mathrm{~g}$ of ASPB was firstly added into $45 \mathrm{~mL}$ mixture of water and ethanol (v/v, 2/1) with ultrasonic dispersion for $20 \mathrm{~min}$ followed by addition of $1.34 \mathrm{~g}$ of distilled Py $(0.2 \mathrm{~mol})$. After the mixture was cooled to $5^{\circ} \mathrm{C}$ and degassed under $\mathrm{N}_{2}$ flow for $15 \mathrm{~min}, 2.7 \mathrm{~g}(0.1 \mathrm{~mol})$ of $\mathrm{FeCl}_{3} \cdot 6 \mathrm{H}_{2} \mathrm{O}$ in $15 \mathrm{~mL}$ water aqueous solution was added, and the reaction was allowed to continue for $6 \mathrm{~h}$. The resulting products were then collected via filtration, and were subjected to three sequential washings with ethanol and distilled water before finally being dried in vacuum at $60^{\circ} \mathrm{C}$ for $12 \mathrm{~h}$.

\subsection{Characterization methods}

\subsubsection{Morphological and structural analysis}

Morphological studies were carried out using a Quanta 200 (FEI Company, Netherlands) scanning electron microscope (SEM) operated at $30 \mathrm{kV}$. FTIR spectra for PPy and PPy/ASPB nanocomposite were obtained using a Nicolet AVATAR 360FT spectrometer (Thermo Nicolet Corporation, USA). Raman spectra were obtained with a RW-1000 system (Renishaw Company, London, United Kingdom) using a $\mathrm{Ar}^{+}$laser operating at $514.5 \mathrm{~nm}$ with a $\mathrm{CCD}$ detector. X-Ray diffraction (XRD) measurements were carried out on a Shimadzu 'XRD-6000' instrument (Kyoto, Japan) operating at a voltage of $40 \mathrm{kV}$ and a current of $40 \mathrm{~mA}$ with $\mathrm{CuK}_{\alpha}$ radiation $(\lambda=1.54060 \AA)$. The samples were measured in a continuous scan mode at $10-60^{\circ}(2 \theta)$ with a scanning rate of $5 \% \mathrm{~min}$. XPS spectra were recorded using a VGESCALAB MK II system (United Kingdom) operated in the constant analyzer energy mode.

\subsubsection{Electrical conductivity and thermal analysis}

TGA was performed on a SETSYS-1750 instrument at a heating rate of $10^{\circ} \mathrm{C} / \mathrm{min}$ in $\mathrm{N}_{2}$ atmosphere. The electrical conductivities of the samples were measured in a four-point probe (RTS-4, China) apparatus at room temperature. After the samples were pressed into the circular tablet with $D=13 \mathrm{~mm}$ at $20 \mathrm{MPa}$, the thickness $W$ of each tablet was measured. Then $F(W / S)$ and $F(D / S)(S=1)$ were looked up from a table to calculate the source current $I$ according to formula (1). $F(W / S)$ denoted the width correction coefficient, and $F(D / S)$ the diameter correction coefficient. Since conductivity $(\sigma$; $\mathrm{S} / \mathrm{cm})=1 / \rho$, the electrical conductivities of resulting products could be calculated by Equation (1):

$I=F(W / S) \cdot F(D / S) \cdot W \cdot 0.1$ 


\section{Results and discussion}

\subsection{Morphologies}

Figure 1 displays the morphologies of samples, and the schematic representations of formation mechanisms are shown in Figure 2. As shown by the SEM images, PPy displays a typically cauliflower-like or tumor-like structure (Figure 1a, b). The performance of three-dimensional (3D) polymer growth can be considered as quasi network systems through the 2, 3 coupling modes as shown in Figure 2a, supported by the 3D VRH (Variable range hopping) for $\sigma_{\mathrm{dc}}(T)$ [23]. The interchain links or side chains through the 2, 3 coupling modes can lead to the poor solubility of PPy in organic solvents [24]. The addition of ASPB with uniform spherical structure (Figure 1d) provides the space factors for Py orderly growth (Figure $2 b$ ), resulting in special morphology of PPy. As shown in Figure 1c, PPy/ASPB nanocom- posite shows sphere-like structure and the particle size is much less than that of PPy (Figure 1a). Therefore, the SEM results elucidate that the reactions are remarkably effective for the PPy functionalization.

\subsection{FTIR and Raman spectra studies}

FTIR spectra of samples are represented in Figure 3. The FTIR spectrum of PPy shows the characteristic bands attributable to the $\mathrm{C}-\mathrm{H}$ in-plane deformation vibration at $1039 \mathrm{~cm}^{-1}, \mathrm{C}-\mathrm{C}$ asymmetric stretching vibration at $1458 \mathrm{~cm}^{-1}$, ring-stretching mode of Py ring at $1540 \mathrm{~cm}^{-1}$ [25]. These characteristic peaks from PPy are clearly shown in the FTIR spectrum of PPy/ASPB nanocomposite (1036, 1451 and $1546 \mathrm{~cm}^{-1}$ ). In addition, PPy/ASPB nanocomposite shows some new bands: the broad band at $1292 \mathrm{~cm}^{-1}$ is attributed to $\mathrm{C}-\mathrm{H}$ and $\mathrm{C}-\mathrm{N}$ in-plane deformation

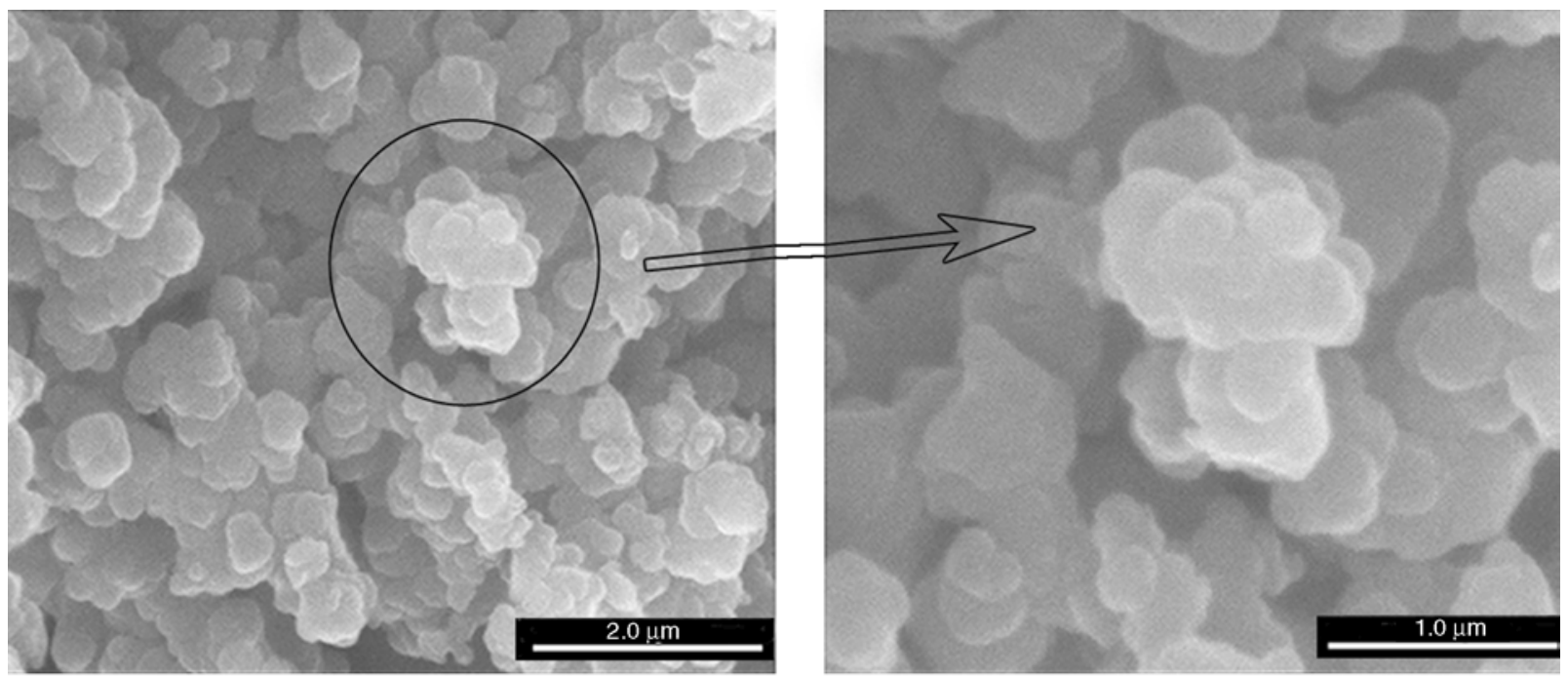

a)

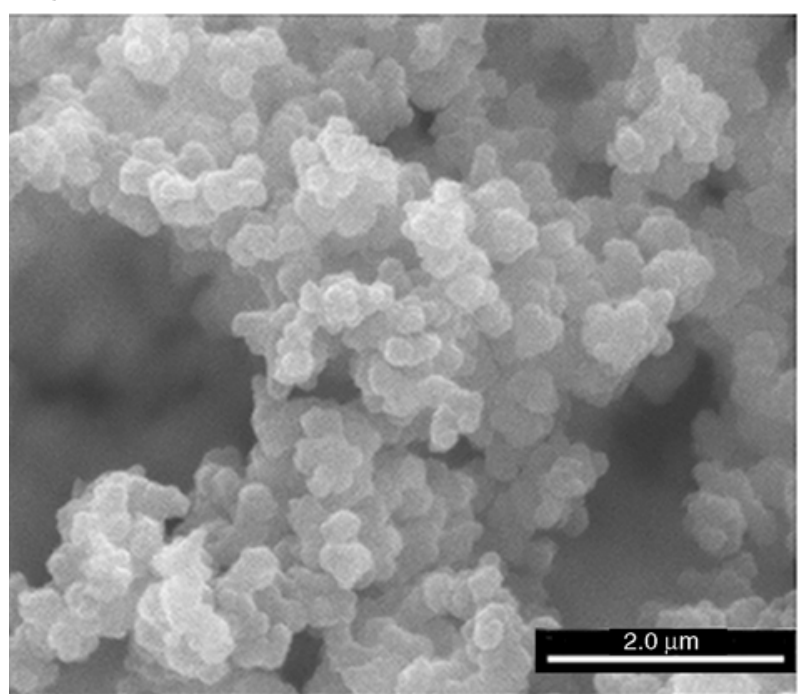

c) b)

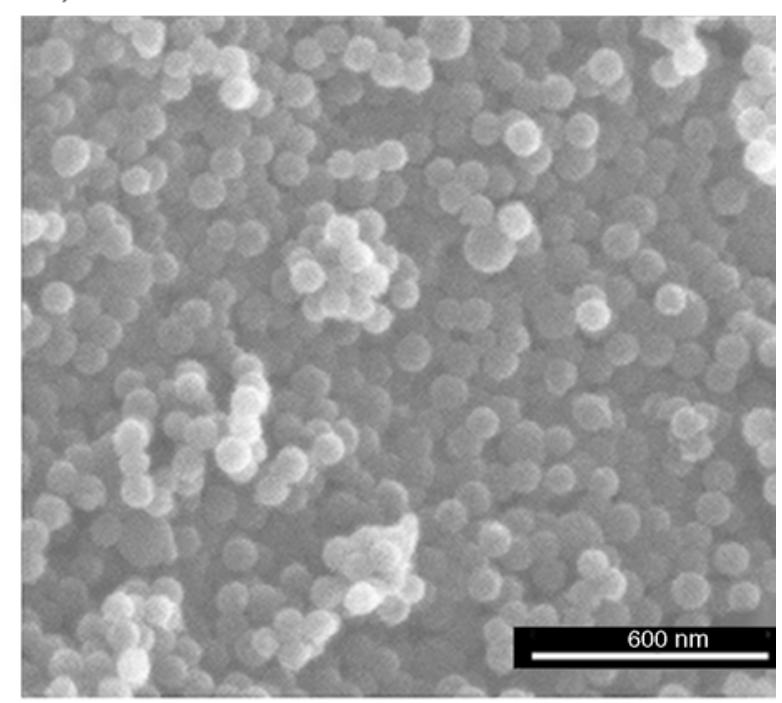

d)

Figure 1. SEM images of PPy (a, b), PPy/ASPB nanocomposite (c) and ASPB (d) 


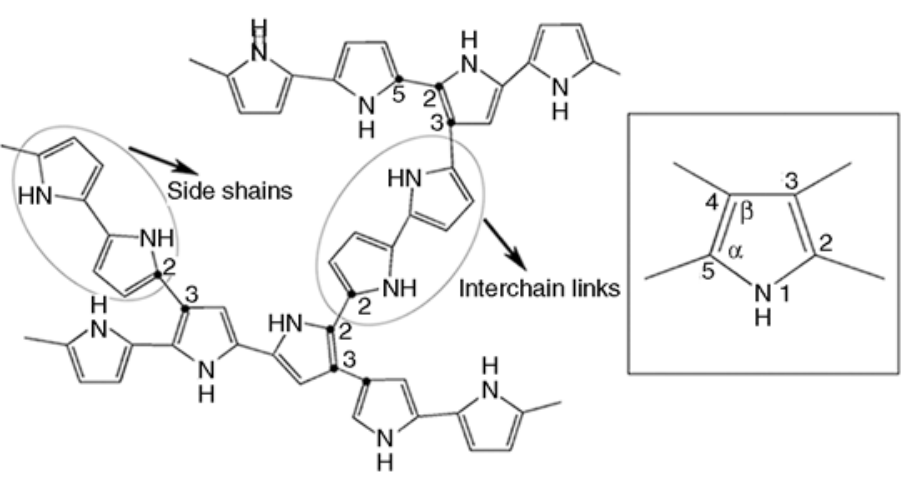

a)

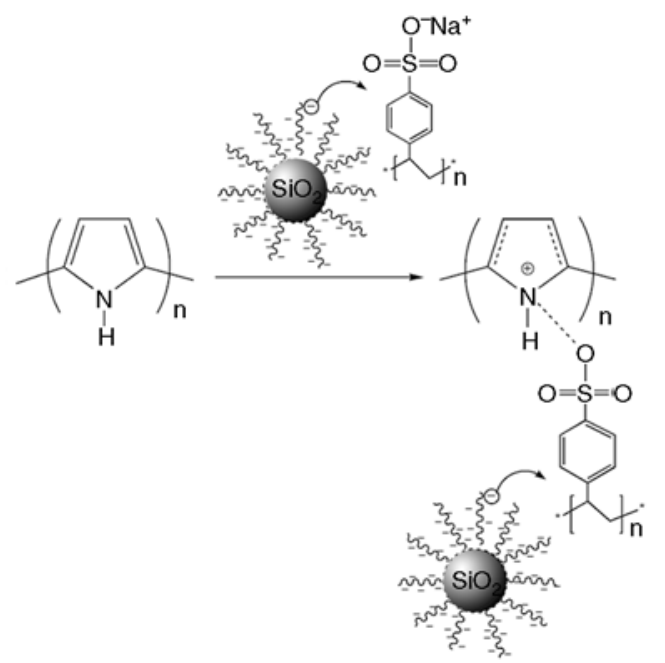

b)

Figure 2. Schematic representations of formation mechanisms of PPy (a) and PPy/ASPB nanocomposite (b)

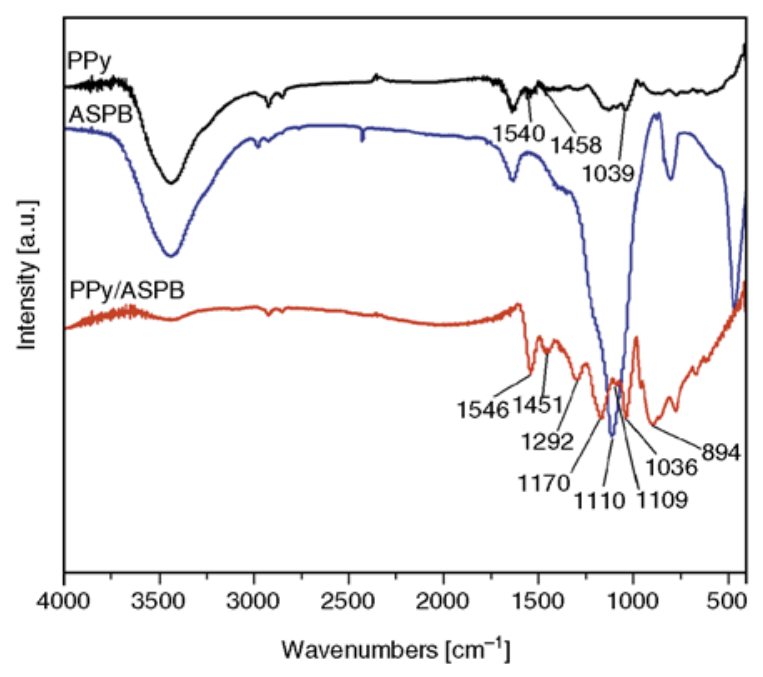

Figure 3. FTIR spectra of PPy, ASPB, and PPy/ASPB nanocomposite

vibrations; the strong bands at 1170 and $894 \mathrm{~cm}^{-1}$ are characteristic of doped PPy chains [11]. The peak at $1109 \mathrm{~cm}^{-1}$ may be attributed to $\mathrm{Si}-\mathrm{O}-\mathrm{Si}$ stretching vibrations, which appears at $1110 \mathrm{~cm}^{-1}$ in the spectrum of ASPB, suggesting that ASPB have already been doped in PPy system. In addition, the ratio of the integrated absorption areas of the 1451 and $1546 \mathrm{~cm}^{-1}\left(A_{1451} / A_{1546}\right)$ bands for the PPy/ASPB nanocomposite is 0.38 , which is higher than that of $\operatorname{PPy}\left(A_{1458} / A_{1540}=0.27\right)$. This suggests the conjugation length increases [17].

Figure 4 shows the Raman spectra of PPy and PPy/ ASPB nanocomposite. The prominent bands that emerged in the spectrum of PPy can also be observed in the spectrum of PPy/ASPB nanocomposite. The appearance of peak at $1055 \mathrm{~cm}^{-1}\left(1060 \mathrm{~cm}^{-1}\right.$ for

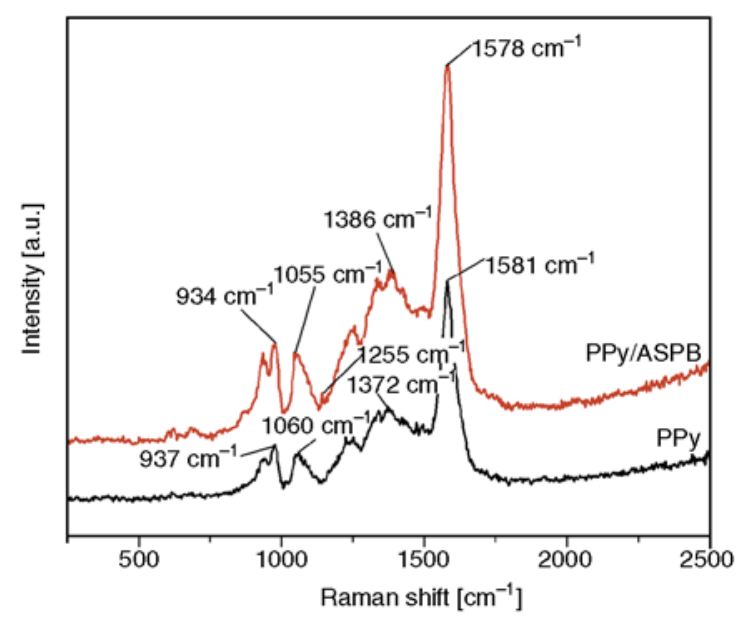

Figure 4. Raman spectra of PPy and PPy/ASPB nanocomposite

PPy) has been associated with the quinonoid polaron structure and those at $934 \mathrm{~cm}^{-1}\left(937 \mathrm{~cm}^{-1}\right.$ for PPy) and $1255 \mathrm{~cm}^{-1}$ (no presence in PPy spectrum) have been associated with the quinonoid bipolaron structure, revealing the presence of doped PPy structures $[6,26]$. The peak at $1578 \mathrm{~cm}^{-1}\left(1581 \mathrm{~cm}^{-1}\right.$ for PPy $)$ attributable to $\mathrm{C}=\mathrm{C}$ stretching is considered to be an overlap of two oxidized structures. Therefore, compared with the PPy, PPy/ASPB nanocomposite has an increased portion of bipolarons $\left(1255 \mathrm{~cm}^{-1}\right)$ relative to polarons in the Raman spectrum, leading to the high conductivity [27].

\subsection{Crystallographic structure}

The crystallographic structures of samples are characterized using XRD measurements. As shown in Figure 5, PPy only exhibits a broad characteristic 


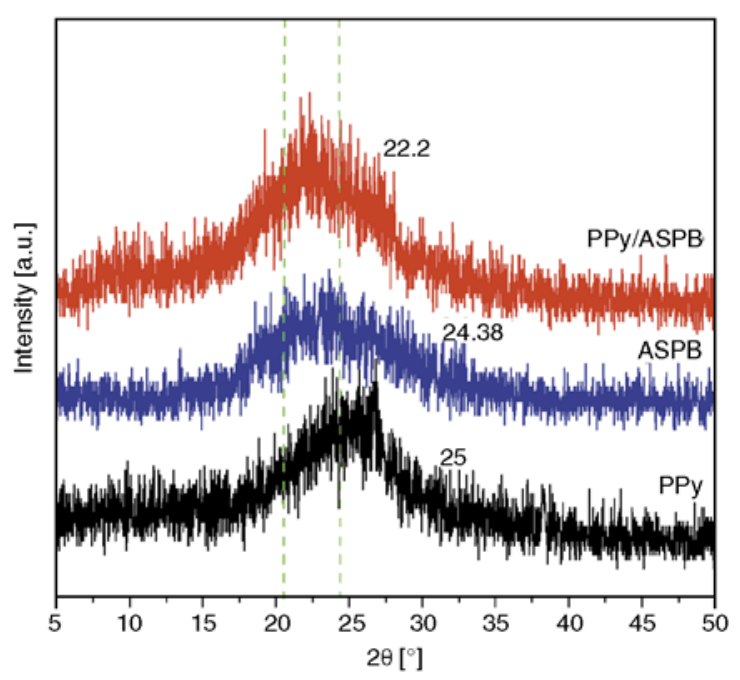

Figure 5. XRD patterns of PPy, ASPB and PPy/ASPB nanocomposite

peak at $2 \theta=25^{\circ}$, implying an amorphous structure [28]. For the XRD pattern of ASPB, the characteristic peak at $2 \theta=24.38^{\circ}$ appears, corresponding to $\mathrm{SiO}_{2}$ reflection peak. For the PPy/ASPB nanocomposite, the broad characteristic peak has shifted to $22.2^{\circ}$. The changes may be due to the doping of
ASPB ( $\mathrm{SiO}_{2}$ cores) into conducting polymer matrix. In addition, full width at half maximum (FWHM) of PPy/ASPB nanocomposite $\left(10.1^{\circ}\right)$ is higher than that of PPy $\left(7.6^{\circ}\right)$, which confirms that the crystallinity of PPy/ASPB nanocomposite is decreased, which helps to the increase of solubility.

\subsection{XPS study}

Wide region spectroscopy of PPy and PPy/ASPB nanocomposite are shown in Figure 6a. The narrow range spectra of $\mathrm{C}_{1 \mathrm{~s}}, \mathrm{O}_{1 \mathrm{~s}}$ and $\mathrm{N}_{1 \mathrm{~s}}$ of PPy/ASPB nanocomposite are also depicted (Figure 6b-6d). Compared with the wide region spectroscopy of PPy, the peaks at 101.9 and $167.3 \mathrm{eV}$ for PPy/ASPB nanocomposite spectrum have been observed, suggesting the existence of Silicon and Sulfur signals respectively (from ASPB).

For the chemically synthesized PPy/ASPB nanocomposite, the $\mathrm{C}_{1 \mathrm{~s}}$ main peak is decomposed into five lines (Figure 6b). The lowest binding energy centered peak (main C peak) at $284.1 \mathrm{eV}$ corresponds to the $\beta$-carbons of Py ring and covalent bonded carbon in the ASPB dopant, while the binding energy cen-

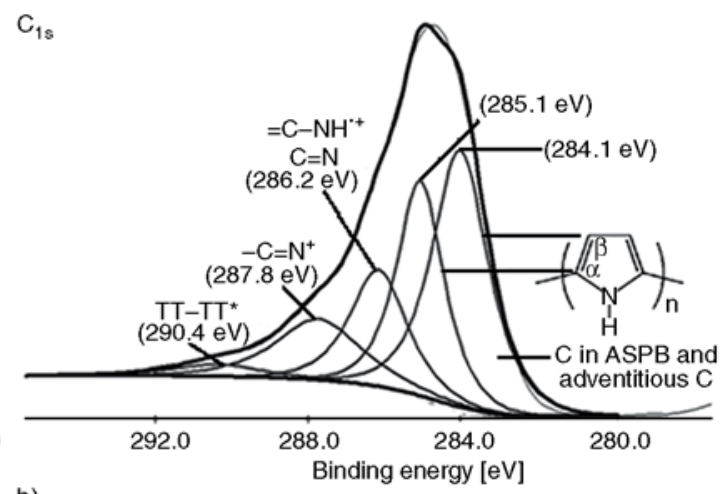

b)

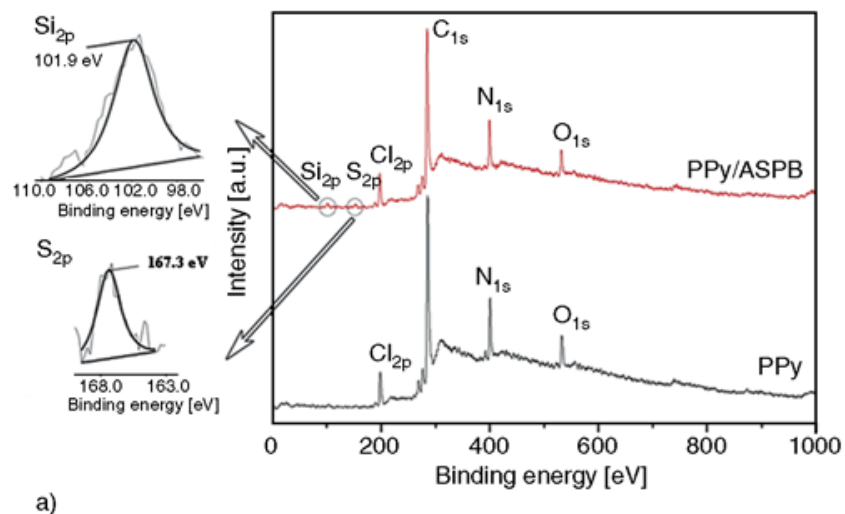

a)

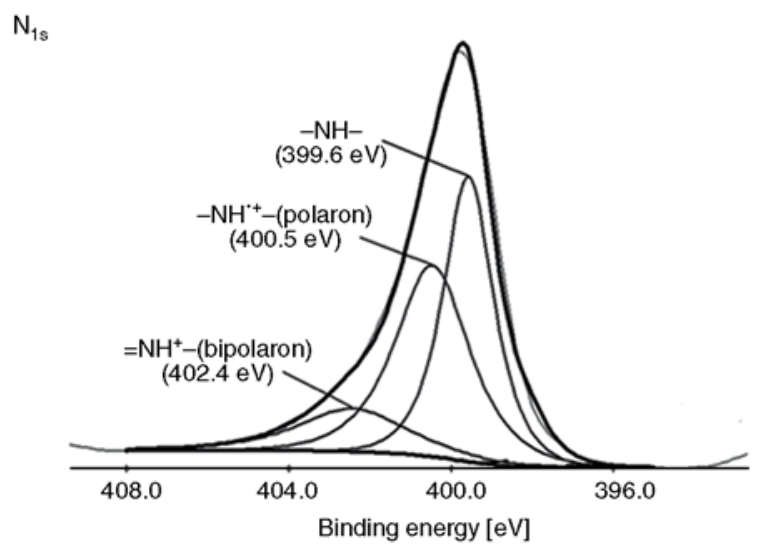

c)

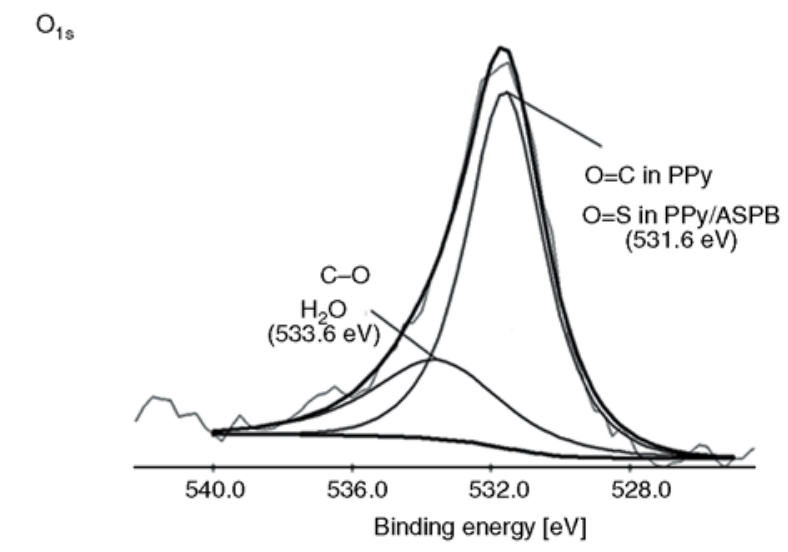

d)

Figure 6. XPS spectra of (a) wide region spectroscopy, (b) $\mathrm{C}_{1 \mathrm{~s}}$, (c) $\mathrm{N}_{1 \mathrm{~s}}$, (d) O1s of PPy/ASPB nanocomposite 
tered peak at $285.1 \mathrm{eV}$ corresponds to $\alpha$ - carbons. Three other peaks are identified by the deconvolution of the signal at 286.2, 287.8 and $290.4 \mathrm{eV}$, respectively belonging to the defects in doped PPy. According to the literature by Ruangchuay et al. [29], the peak at $286.2 \mathrm{eV}$ is contributed by the imine-like $(\mathrm{C}=\mathrm{N})$, polaron $\left(=\mathrm{C}-\mathrm{NH}^{\cdot+}\right)$, hydroxylated defects $(\mathrm{C}-\mathrm{OH})$ and $\mathrm{C}-\mathrm{S}$ from the dopant. The peak positioned at $287.8 \mathrm{eV}$ is associated to the bipolarons $\left(-\mathrm{C}=\mathrm{N}^{+}\right)$and carbonylic defects $(\mathrm{C}=\mathrm{O})$. The highest binding energy peak at $290.4 \mathrm{eV}$ can be assigned to $\pi-\pi^{*}$ satellite commonly found in aromatic PPy, $6.3 \mathrm{eV}$ higher than that of the main $\mathrm{C}$ peak.

As shown in Figure 6c, the deconvolution of $\mathrm{N}_{1 \mathrm{~s}}$ signals in the XPS spectrum gives three Gaussian components. The main $\mathrm{N}$ peak at $399.6 \mathrm{eV}$ is attributed to the neutral $\mathrm{N}$ in the Py ring $(-\mathrm{NH}-)$. The imine-like nitrogen $(=\mathrm{N}-)$ is disappeared while two new peaks at high binding energy are present [30]. The high binding energy peak can be attributed to oxidized/protonated N. Comparing to the position of the main peak, the $0.9 \mathrm{eV}$ higher binding energy $(400.5 \mathrm{eV})$ peak is indicative of $-\mathrm{NH}^{\cdot+}-$ in the polaron charge carrier species, while the $2.8 \mathrm{eV}$ higher binding energy $(402.4 \mathrm{eV})$ peak can be assigned to $=\mathrm{NH}^{+}-$, a bipolaron charge carrier species [31]. Upon consideration of the literature, the nitrogen signal of dedoped PPy only has three peaks at about $397.8,399.7$, and $400.5 \mathrm{eV}$. The present set of peaks is shifted to higher binding energy compared to that of PPy.

The oxygen signal $\left(\mathrm{O}_{1 \mathrm{~s}}\right)$ in the XPS spectrum is shown in Figure 6d. Two peaks at 531.6 and $533.6 \mathrm{eV}$ are found from the deconvolution of the signal. The peak at $531.6 \mathrm{eV}$ can be attributed to the $\mathrm{O}=\mathrm{S}$ of the dopant present in the PPy/ASPB nanocomposite [32], while the peak located at $533.6 \mathrm{eV}$ may be related to the oxygen contribution of bound water and polymer-oxidized moieties.

XPS studies indicate that doping of ASPB into PPy matrix is achieved, as expected, which is consistent with both FTIR studies.

\subsection{Measurements of electrical conductivity}

The electrical conductivities of PPy and PPy/ASPB nanocomposite are determined using a RTS-4 four point probe resistivity measurement system. Results indicate that the room temperature electrical conductivity of PPy is $3.6 \mathrm{~S} / \mathrm{cm}$, however, PPy/ASPB nanocomposite shows a high value of conductivity $(20 \mathrm{~S} / \mathrm{cm})$. Also, it is higher than those reported by literatures, including PPy-grafted multiwalled carbon nanotube (MWCNT) [25], polypropylene (PP)/ montmorillonite (MMT)/PPy nanocomposites [33], $\mathrm{PPy} / \mathrm{SiO}_{2}$ nanocomposite [34] and PPy/PSS coreshell nanoparticles [35]. Increase in magnitude of conductivity is consistent with the FTIR and Raman spectra results.

\subsection{Thermal gravimetric analysis}

Figure 7 displays the thermal gravimetric analysis of PPy, ASPB and PPy/ASPB nanocomposite under $\mathrm{N}_{2}$ and $\mathrm{O}_{2}$ atmospheres at $60 \%$ RH. As observed from the Figure $7 \mathrm{a}$, it demonstrates that the thermal stability of ASPB is much higher than that of PPy in the temperature range of $35-750^{\circ} \mathrm{C}$. As PPy is hygroscopic, nearly $7.9 \mathrm{wt} \%$ weight loss has occurred at $100^{\circ} \mathrm{C}$, due to the evaporation of residual water while only $2 \mathrm{wt} \%$ weight loss for PPy/ASPB nanocomposite. As the temperature increases, the main mass loss of PPy and PPy/ASPB nanocomposite starts at about $250^{\circ} \mathrm{C}$ corresponding to PPy degradation [36]. In case of PPy, the weight reduces largely with only one endothermic peak at $286^{\circ} \mathrm{C}$ observed in Figure 7b. However, a considerable enhancement in thermal stability has been seen for PPy/ASPB nanocomposite. Finally at $600^{\circ} \mathrm{C}$, weight loss for PPy is almost $100 \%$, much more than that for PPy/ASPB nanocomposite (25\%). The increase of PPy/ASPB nanocomposite residual content can also be displayed in Figure 7c. Compared with the DSC curve of PPy at $\mathrm{N}_{2}$ atmosphere (Figure 7b), the curve of PPy under $\mathrm{O}_{2}$ atmosphere (Figure 7d) shows the end of a sharp exothermic peak at $475.5^{\circ} \mathrm{C}$ because of additional oxygen involving in the reaction [37]. For the DSC curve of PPy/ASPB nanocomposite, the exothermic peaks are not obvious and move to the high temperature. All the results show that introducing ASPB into the PPy system can enhance the thermal stability of PPy. The improvement in the thermal stability can also be attributed to the increase in orderliness of PPy chains, supported by analysis of XRD, SEM and conductivity measurements. 

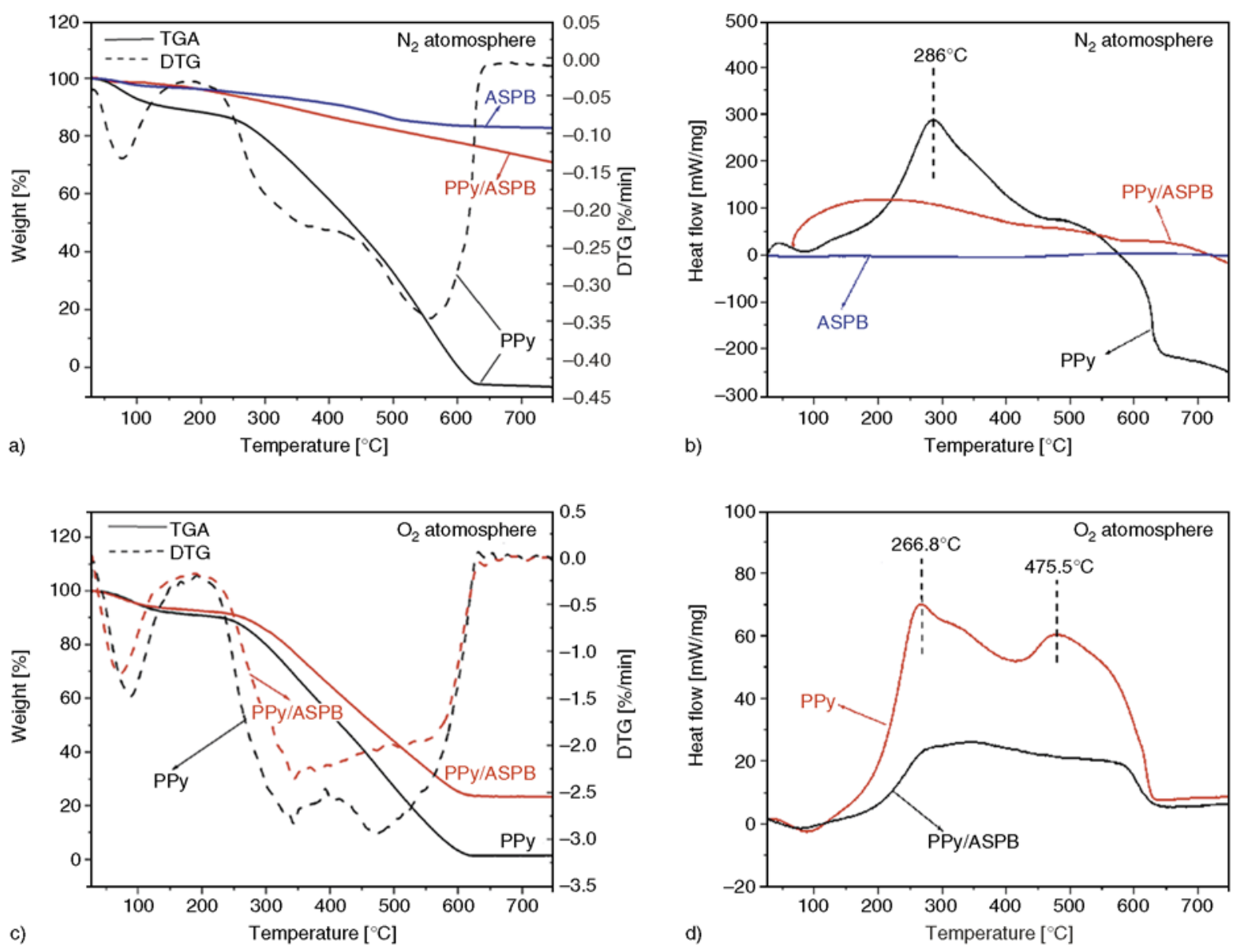

Figure 7. Thermal gravimetric analysis of PPy, ASPB and PPy/ASPB nanocomposite under $\mathrm{N}_{2}$ atmosphere (a, b) and under $\mathrm{O}_{2}$ atmosphere $(\mathrm{c}, \mathrm{d})$

\section{Conclusions}

In summary, novel PPy/ASPB nanocomposite by means of in situ chemical oxidation polymerization method has been described. Different characterization and analytical methods confirm that ASPB is an excellent dopant for PPy. Compared with PPy, PPy/ASPB nanocomposite possesses the appearance of sphere-like morphology, enhanced thermal stability and higher room temperature electrical conductivity. Therefore, for the doped PPy system, more work is certainly necessary to validate the above treatment for other intrinsically conducting polymers.

\section{Acknowledgements}

This research was supported by Scientific Research Program of Wuhan University (217274815) and National Natural Science Foundational of China (31170558).

\section{References}

[1] Shirakawa H., Louis E. J., MacDiarmid A. G., Chiang C. K., Heeger A. J.: Synthesis of electrically conducting organic polymers: Halogen derivatives of polyacetylene, $(\mathrm{CH})_{\mathrm{x}}$. Journal of the Chemical Society, Chemical Communications, 16, 578-580 (1977).

DOI: 10.1039/C39770000578

[2] Jang J., Nam Y., Yoon H.: Fabrication of polypyrrolepoly(N-vinylcarbazole) core-shell nanoparticles with excellent electrical and optical properties. Advanced Materials, 17, 1382-1386 (2005).

DOI: $10.1002 /$ adma.200401841

[3] Wu T-M., Lin S-H.: Synthesis, characterization, and electrical properties of polypyrrole/multiwalled carbon nanotube composites. Journal of Polymer Science Part A: Polymer Chemistry, 44, 6449-6457 (2006). DOI: $10.1002 /$ pola.21724

[4] Carrasco P. M., Grande H. J., Cortazar M., Alberdi J. M., Areizaga J., Pomposo J. A.: Structure-conductivity relationships in chemical polypyrroles of low, medium and high conductivity. Synthetic Metals, 156, 420-425 (2006).

DOI: $10.1016 /$ j.synthmet.2006.01.005 
[5] DeArmitt C., Armes S. P.: Colloidal dispersions of surfactant-stabilized polypyrrole particles. Langmuir, $\mathbf{9}$, 652-654 (1993).

DOI: $10.1021 / 1 \mathrm{a} 00027 \mathrm{a} 007$

[6] Yuan X., Zeng X., Zhang H-J., Ma Z-F., Wang C-Y.: Improved performance of proton exchange membrane fuel cells with $p$-toluenesulfonic acid-doped Co-PPy/C as cathode electrocatalyst. Journal of the American Chemical Society, 132, 1754-1755 (2010).

DOI: $10.1021 / \mathrm{ja9} 09537 \mathrm{~g}$

[7] Lee J. Y., Kim D. Y., Kim C. Y.: Synthesis of soluble polypyrrole of the doped state in organic solvents. Synthetic Metals, 74, 103-106 (1995).

DOI: 10.1016/0379-6779(95)03359-9

[8] Lee J. Y., Song K. T., Kim S. Y., Kim Y. C., Kim D. Y., Kim C. Y.: Synthesis and characterization of soluble polypyrrole. Synthetic Metals, 84, 137-140 (1997). DOI: 10.1016/S0379-6779(97)80683-2

[9] Oh E. J., Jang K. S., Suh J. S., Kim H., Kim K. H., Yo C. H., Joo J.: UV-Vis./NIR and transport studies of chemically synthesized soluble polypyrrole. Synthetic Metals, 84, 147-148 (1997).

DOI: $10.1016 / \mathrm{S} 0379-6779(97) 80686-8$

[10] Shen Y., Wan M.: In situ doping polymerization of pyrrole with sulfonic acid as a dopant. Synthetic Metals, 96, 127-132 (1998).

DOI: $10.1016 / \mathrm{S} 0379-6779(98) 00076-9$

[11] Wu T-M., Chang H-L., Lin Y-W.: Synthesis and characterization of conductive polypyrrole with improved conductivity and processability. Polymer International, 58, 1065-1070 (2009).

DOI: $10.1002 /$ pi.2634

[12] Cairns D. B., Armes S. P., Chehimi M. M., Perruchot C., Delamar M.: X-ray photoelectron spectroscopy characterization of submicrometer-sized polypyrrolepolystyrene composites. Langmuir, 15, 8059-8066 (1999).

DOI: $10.1021 / 1 \mathrm{a99} 9443 \mathrm{k}$

[13] Jaramillo A., Spurlock L. D., Young V., Brajter-Toth A.: XPS characterization of nanosized overoxidized polypyrrole films on graphite electrodes. Analyst, 124, 1215-1221 (1999).

DOI: $10.1039 / A 902578 B$

[14] Ruangchuay L., Schwank J., Sirivat A.: Surface degradation of $\alpha$-naphthalene sulfonate-doped polypyrrole during XPS characterization. Applied Surface Science, 199, 128-137 (2002).

DOI: 10.1016/S0169-4332(02)00564-0

[15] Nicho M. E., Hu H.: Fourier transform infrared spectroscopy studies of polypyrrole composite coatings. Solar Energy Materials and Solar Cells, 63, 423-435 (2000).

DOI: $10.1016 / \mathrm{S} 0927-0248(00) 00061-1$
[16] Ekramul Mahmud H. N. M., Kassim A., Zainal Z., Yunus W. M. M.: Fourier transform infrared study of polypyrrole-poly(vinyl alcohol) conducting polymer composite films: Evidence of film formation and characterization. Journal of Applied Polymer Science, 100, 4107-4113 (2006).

DOI: 10.1002/app.23327

[17] Menon V. P., Lei J., Martin C. R.: Investigation of molecular and supermolecular structure in template-synthesized polypyrrole tubules and fibrils. Chemistry of Materials, 8, 2382-3290 (1996).

DOI: $10.1021 / \mathrm{cm} 960203 \mathrm{f}$

[18] Baughman R. H., Shacklelette L. W.: Conductivity as a function of conjugation length: Theory and experiment for conducting polymer complexes. Physical Review B, 39, 5872-5886 (1989).

DOI: 10.1103/PhysRevB.39.5872

[19] Pruneanu S., Graupner W., Oniciu L., Brie M., Turcu R.: Electrochemical and X-ray diffraction studies on polypyrrole films. Materials Chemistry and Physics, 46, 55-60 (1996).

DOI: 10.1016/0254-0584(96)80130-1

[20] Ormond-Prout J., Dupin D., Armes S. P., Foster N. J., Burchell M. J.: Synthesis and characterization of polypyrrole-coated poly(methyl methacrylate) latex particles. Journal of Materials Chemistry, 19, 1433-1442 (2009).

DOI: $10.1039 / \mathrm{b} 816839 \mathrm{c}$

[21] Gu Z., Zhang L., Li C.: Preparation of highly conductive polypyrrole/graphite oxide composites via in situ polymerization. Journal of Macromolecular Science Part B: Physics, 48, 1093-1102 (2009).

DOI: $\underline{10.1080 / 00222340903035576}$

[22] Gao J-W., Li G., Yao Y-F., Jang J-M.: Preparation and characterization of montmorillonite/polypyrrole nanocomposites by in-situ chemical polymerization. Journal of Macromolecular Science Part B: Physics, 50, 1364-1375 (2011).

DOI: $10.1080 / 00222348.2010 .497688$

[23] Joo J., Lee J. K., Baeck J. S., Kim K. H., Oh E. J., Epstein J.: Electrical, magnetic, and structural properties of chemically and electrochemically synthesized polypyrroles. Synthetic Metals, 117, 45-51 (2001). DOI: $10.1016 / \mathrm{S} 0379-6779(00) 00537-3$

[24] Shen Y., Wan M.: Soluble conductive polypyrrole synthesized by in situ doping with $\beta$-naphthalene sulphonic acid. Journal of Polymer Science Part A: Polymer Chemistry, 35, 3689-3695 (1997)

DOI: $10.1002 /($ SICI)1099-0518(199712)35:17<3689:: AID-POLA8>3.0.CO;2-N

[25] Jeon I-Y., Choi H-J., Tan L-S., Baek J-B.: Nanocomposite prepared from in situ grafting of polypyrrole to aminobenzoyl-functionalized multiwalled carbon nanotube and its electrochemical properties. Journal of Polymer Science Part A: Polymer Chemistry, 49, 2529-2537 (2011).

DOI: $10.1002 /$ pola. 24684 
[26] Demoustier-Champagne S., Stavaux P. Y.: Effect of electrolyte concentration and nature on the morphology and the electrical properties of electropolymerized polypyrrole nanotubules. Chemistry of Materials, 11, 829-834 (1999).

DOI: $10.1021 / \mathrm{cm} 9807541$

[27] Gupta S.: Hydrogen bubble-assisted syntheses of polypyrrole micro/nanostructures using electrochemistry: Structural and physical property characterization. Journal of Raman Spectroscopy, 39, 1343-1355 (2008). DOI: $10.1002 / j \mathrm{js} .2002$

[28] Luo Y-L., Fan L-H., Xu F., Chen Y-S., Zhang C-H., Wei Q-B.: Synthesis and characterization of $\mathrm{Fe}_{3} \mathrm{O}_{4} / \mathrm{PPy} /$ $\mathrm{P}(\mathrm{MAA}-\mathrm{co}-\mathrm{AAm})$ trilayered composite microspheres with electric, magnetic and $\mathrm{pH}$ response characteristics. Materials Chemistry and Physics, 120, 590-597 (2010). DOI: 10.1016/j.matchemphys.2009.12.002

[29] Ruangchuay L., Schwank J., Sirivat A.: Surface degradation of $\alpha$-naphthalene sulfonate-doped polypyrrole during XPS characterization. Applied Surface Science, 199, 128-137 (2002).

DOI: $10.1016 / \mathrm{S} 0169-4332(02) 00564-0$

[30] MacDiarmid A. G., Epstein A. J.: Polyanilines: A novel class of conducting polymers. Faraday Discussions of the Chemical Society, 88, 317-332 (1989).

DOI: 10.1039/DC9898800317

[31] Malitesta C., Losito I., Sabbatini L., Zambonin P. G.: New findings on polypyrrole chemical structure by XPS coupled to chemical derivatization labelling. Journal of Electron Spectroscopy and Related Phenomena, 76, 629-634 (1995).

DOI: $10.1016 / 0368-2048(95) 02438-7$
[32] Andreoli E., Rooney D. A., Redington W., Gunning R., Breslin C. B.: Electrochemical deposition of hierarchical micro/nanostructures of copper hydroxysulfates on polypyrrole-polystyrene sulfonate films. The Journal of Physical Chemistry C, 115, 8725-8734 (2011). DOI: $10.1021 /$ jp200465n

[33] Omastová M., Mravčaková M., Chodák I., Pionteck J., Häussler L.: Conductive polypropylene/clay/polypyrrole nanocomposites. Polymer Engineering and Science, 46, 1069-1078 (2006).

DOI: $10.1002 /$ pen.20551

[34] Yuvaraj H., Shim J-J., Lim K. T.: Organic-inorganic polypyrrole-surface modified $\mathrm{SiO}_{2}$ hybrid nanocomposites: A facile and green synthetic approach. Polymers for Advanced Technologies, 21, 424-429 (2010). DOI: $10.1002 /$ pat.1448

[35] Mpoukouvalas K., Wang J., Wegner G.: Conductivity of poly(pyrrole)-poly(styrene sulfonate) core-shell nanoparticles. ChemPhysChem, 11, 139-148 (2010). DOI: $10.1002 /$ cphc. 200900643

[36] Bose S., Kuila T., Uddin M. E., Kim N. H., Lau A. K. T., Lee J. H.: In-situ synthesis and characterization of electrically conductive polypyrrole/graphene nanocomposites. Polymer, 51, 5921-5928 (2010). DOI: $10.1016 /$ j.polymer.2010.10.014

[37] Beck F., Braun P., Oberst M.: Organic electrochemistry in the solid state-overoxidation of polypyrrole. Berichte der Bunsen-Gesellschaft, 91, 967-974 (1987). 